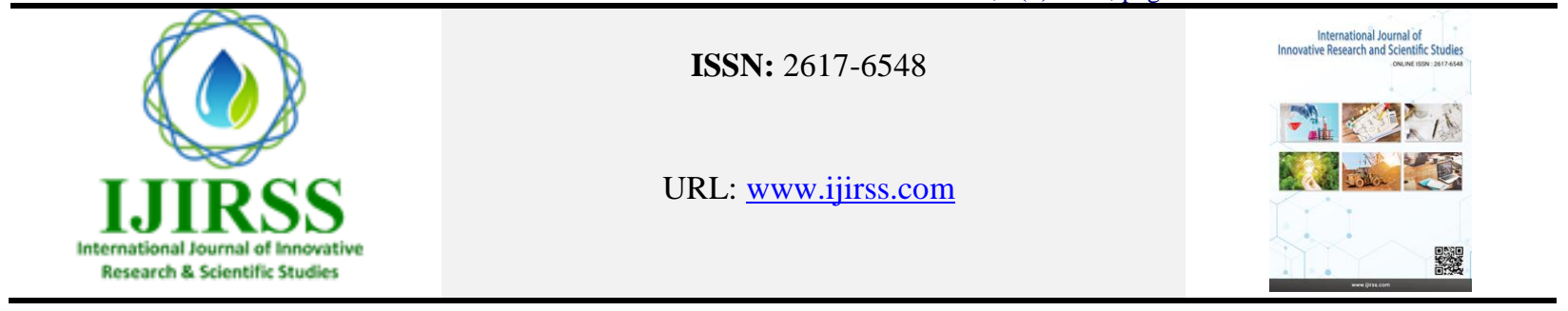

\title{
Evaluation of Solar Radiation for the Use of Photovoltaic (PV) Panels in Afghanistan
}

\author{
Najibullah Hossini \\ Head of Energy Institute of Academy of Science of Afghanistan \\ (Email: h.n.hussaini786@gmail.com)
}

\begin{abstract}
Solar energy is an integral part of living things on Earth, man uses this huge source of energy for various purposes. The sun is very active and is a lingering source of energy for the present and potential for the future. The energy received on the surface of the earth in one year is about 10,000 times the energy consumption of the total population of the world. The use of sunlight in the form of light and heat has been common since ancient times and human beings from the effect of thought and exploration to meet their needs, using the power of reason and experience they have also achieved innovations, innovations and inventions. Using photovoltaic panels to generate solar power in the world, from 2005 to 2015 , it has increased from $5.1 \mathrm{GW}$ to $227 \mathrm{GW}$. The highest amount of solar energy available at noon on summer days, it is approximately equal to $1 \mathrm{KW} / \mathrm{m} 2$, but in most parts of the world this figure is around $200 \mathrm{~W} / \mathrm{m} 2$ on average. The amount of solar radiation energy in Afghanistan, in June, when the angle of the sun shines at a latitude of $23.5^{\circ}$ above the earth, the amount of radiant energy in the southernmost areas of Afghanistan $\left(29.5^{\circ}\right)$, at sunny noon, is equal to $43.70 \mathrm{MJ} / \mathrm{m} 2$ and in December at this width the country will be equal to $(19.85 \mathrm{MJ} / \mathrm{m} 2$. Afghanistan, with its adequate areas and suitable radiation norm $(700 \mathrm{~W} / \mathrm{m} 2)$, has a production capacity of $13548700 \mathrm{MW}$ of electricity.
\end{abstract}

Keywords: Solar Radiation, Fusel Resource, Renewable Resource, Photo Voltaic, Latitude.

DOI: 10.53894 /ijirss.v4i2.69

Funding: This study received no specific financial support.

History: Received: 8 January 2021/Revised: 4 March 2021/Accepted: 24 March 2021/Published: 30 March 2021

Licensed: This work is licensed under a Creative Commons Attribution 4.0 License (cc) $\mathrm{EY}$

Acknowledgement: All authors contributed to the conception and design of the study.

Competing Interests: The authors declare that they have no conflict of interests.

Transparency: The authors confirm that the manuscript is an honest, accurate, and transparent account of the study was reported; that no vital features of the study have been omitted; and that any discrepancies from the study as planned have been explained.

Ethical: This study follows all ethical practices during writing. 


\title{
ارزيابى تشعشع آفتاب جهت استفاده صفحات فوتوولتاييك در شرايط افغانستان نجيب الله حسينى
}

\author{
اكادمى علوم افغانستان و آمر انستبتوت /نرثى، كابل، افغانستان
}

خلاصه

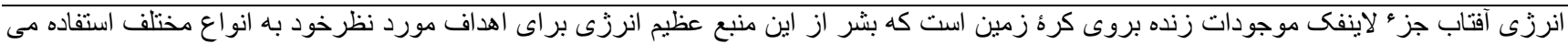

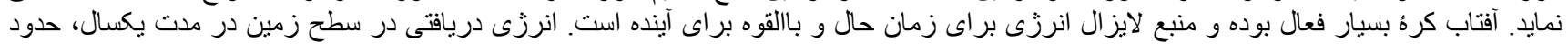

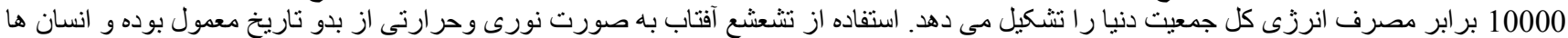

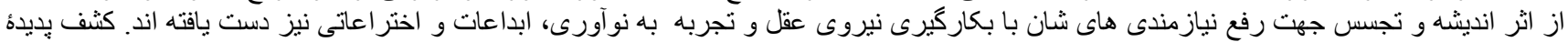

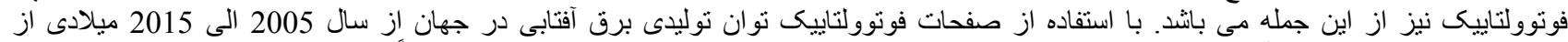

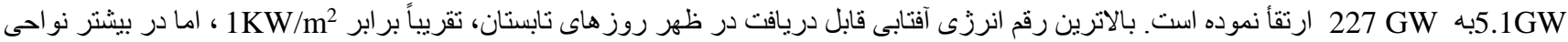

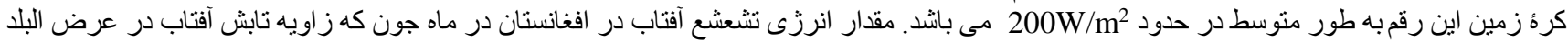

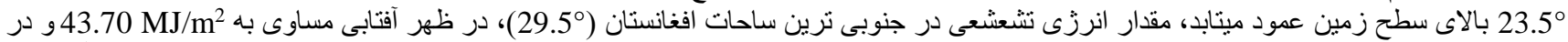

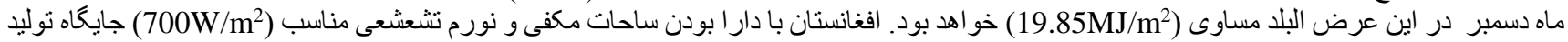
13548700MW

كلمات كليلى : منابع فوسيلى، منابع تجديد يذير، فوتوولتاييك، نشعشع آفتاب، عرض البلد جغر افيايى

1

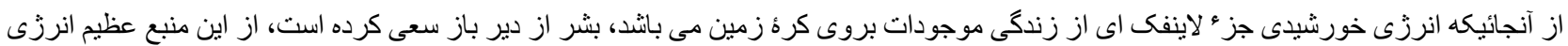

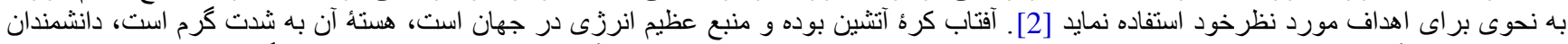

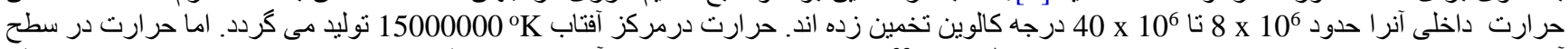

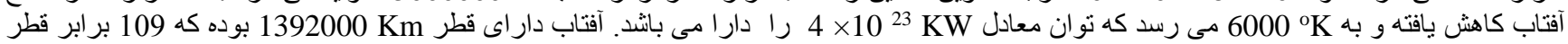

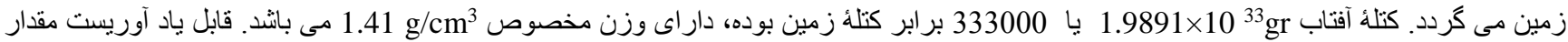

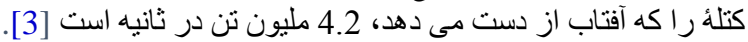

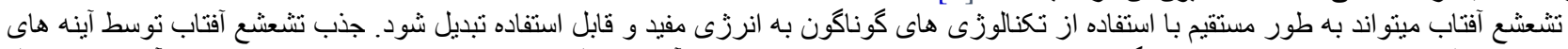

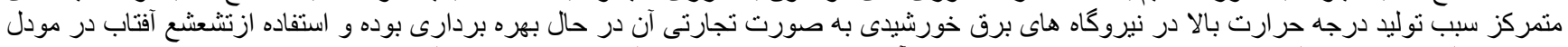

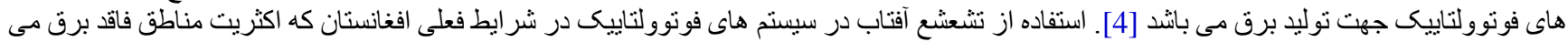

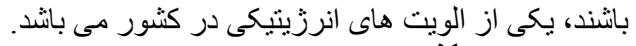

2.

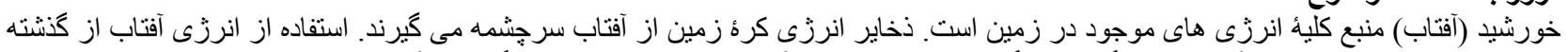

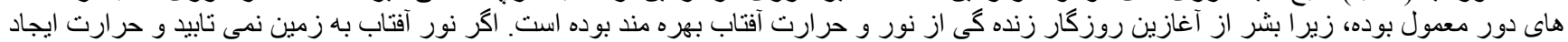

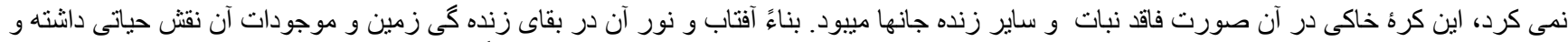

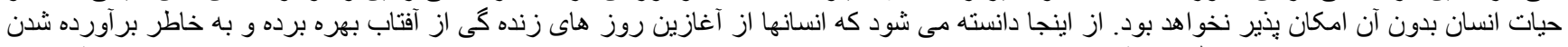

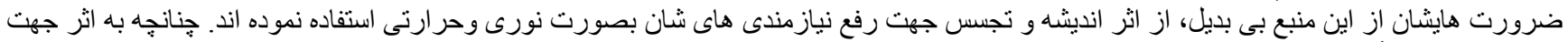

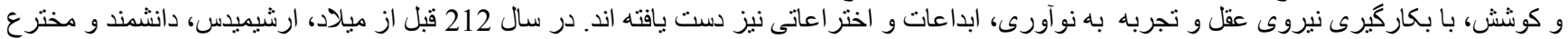

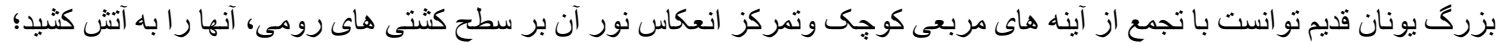

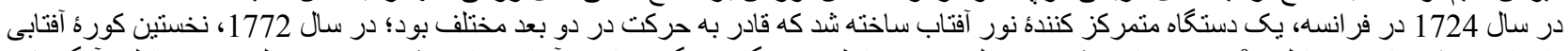

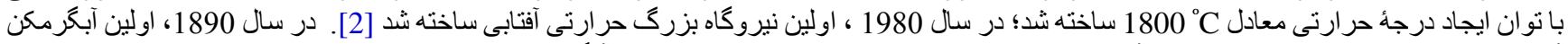

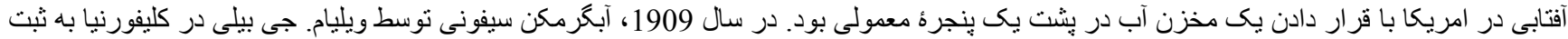

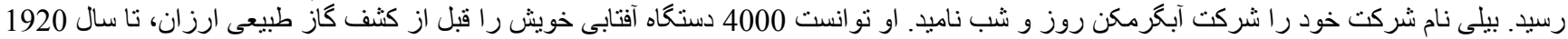

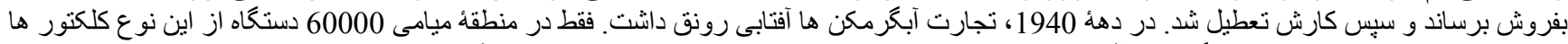

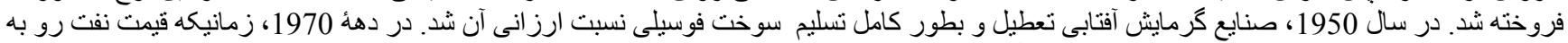

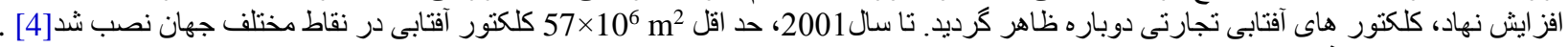

3.

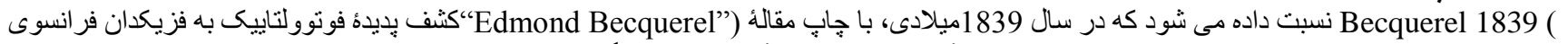

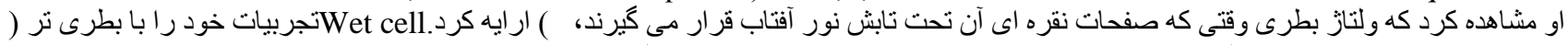

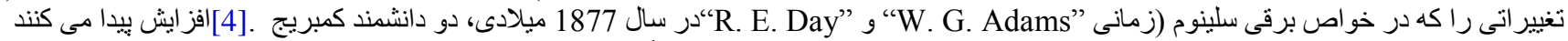

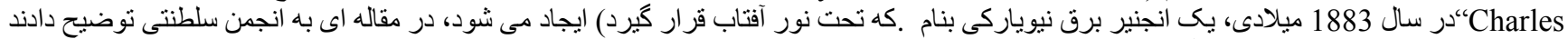

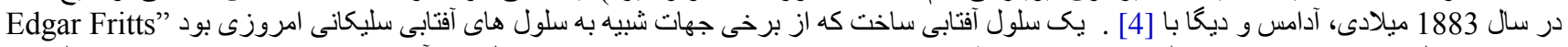

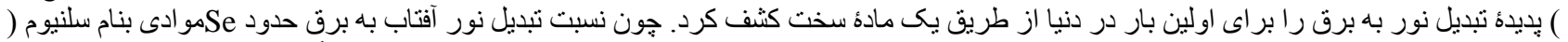

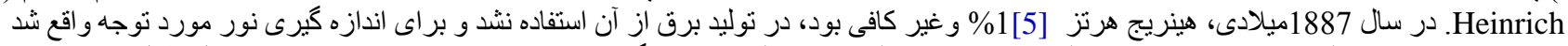

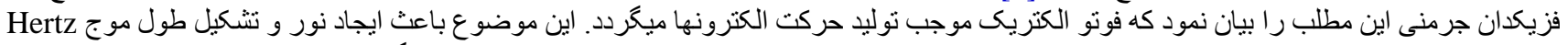

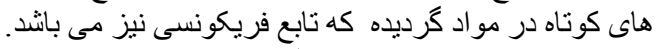

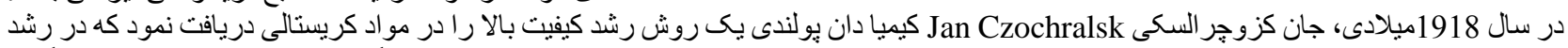

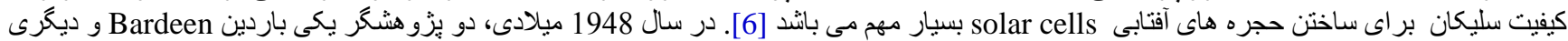

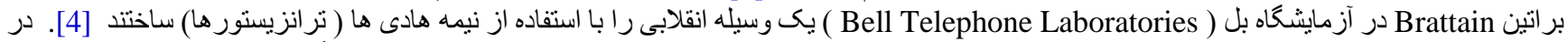

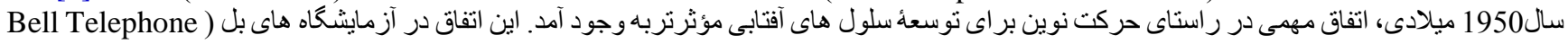


جران (Laboratories

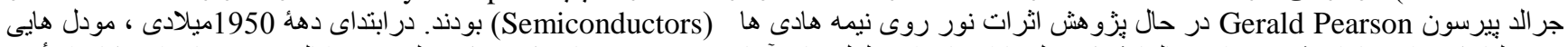

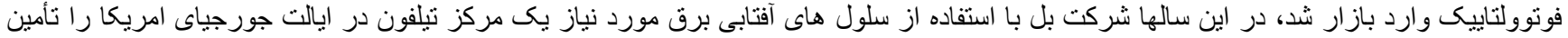

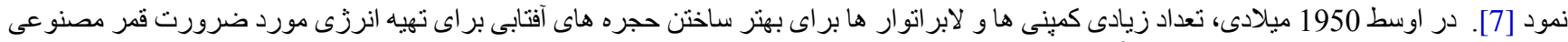

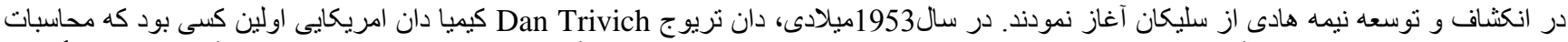

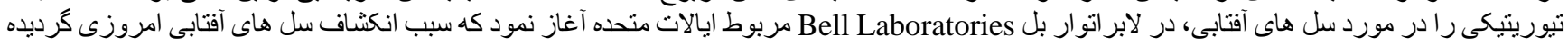

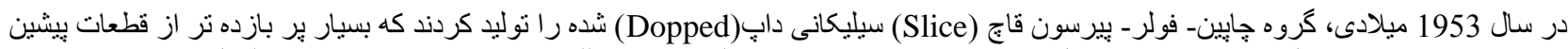

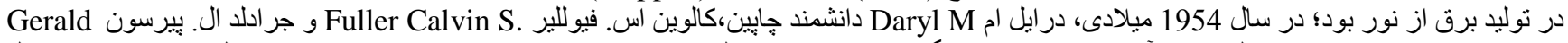

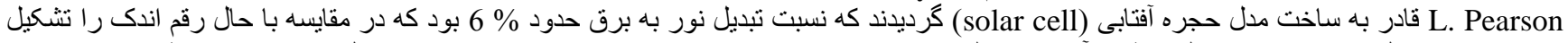

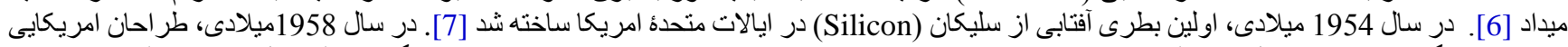

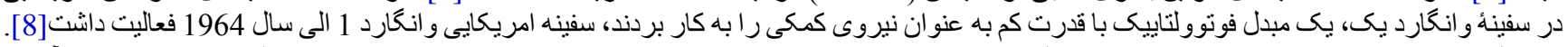

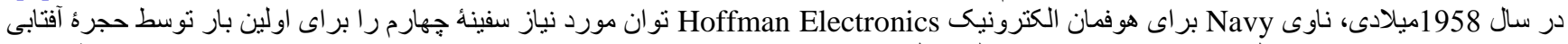

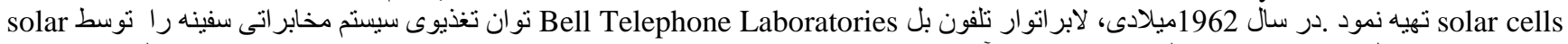

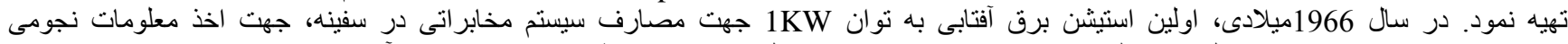

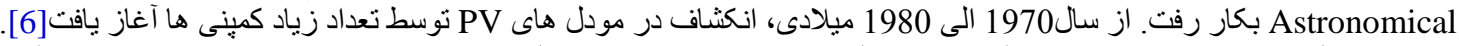

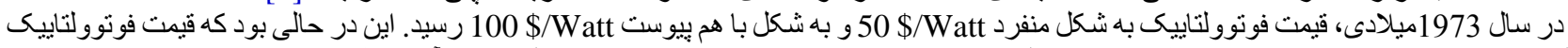

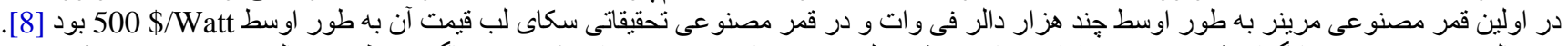

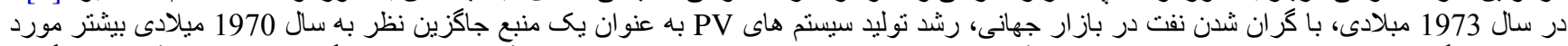

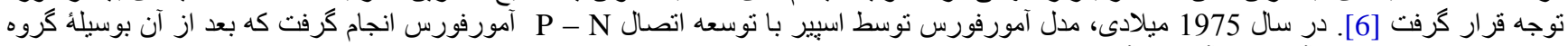

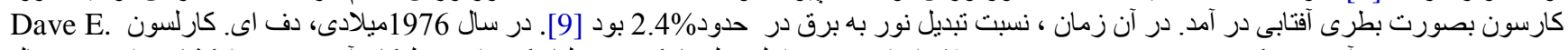

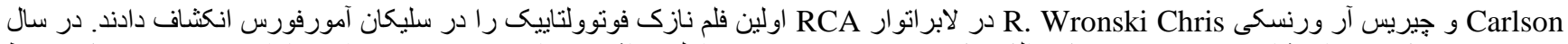

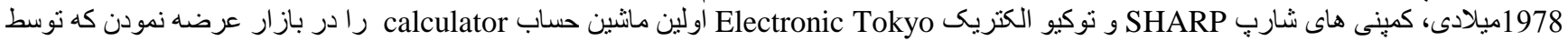

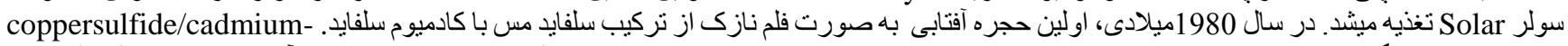

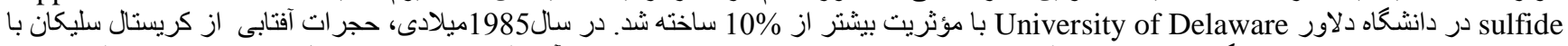

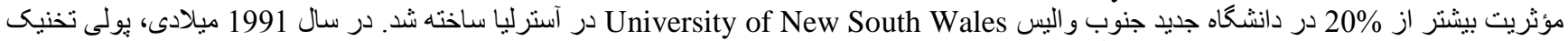

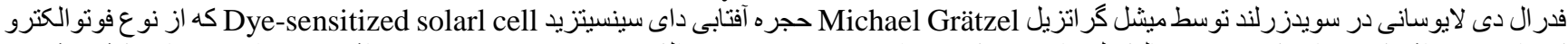

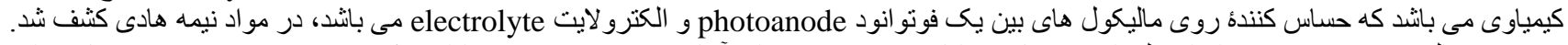

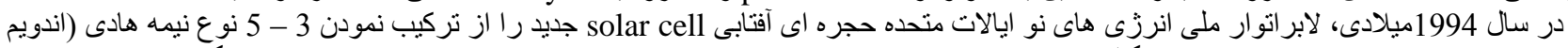

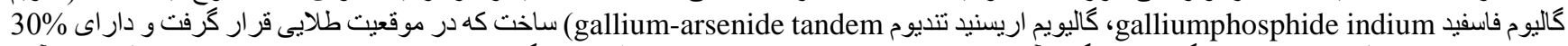

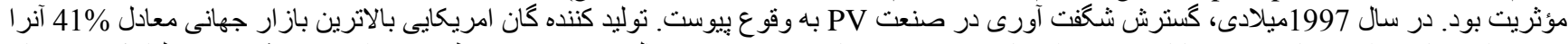

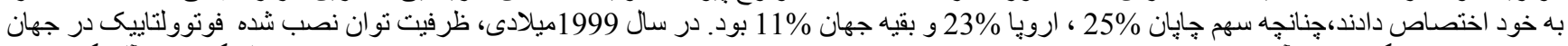

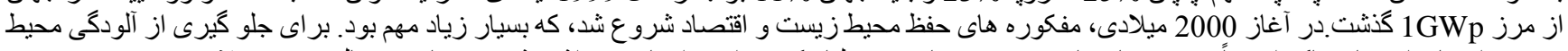

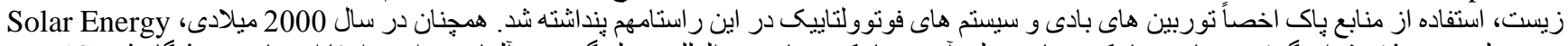

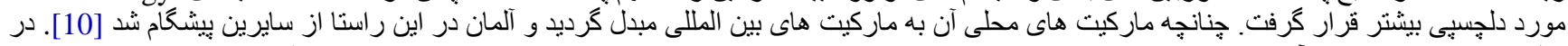

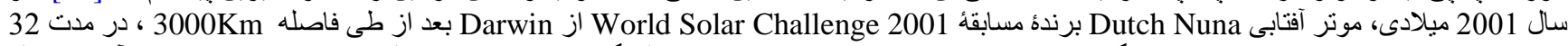

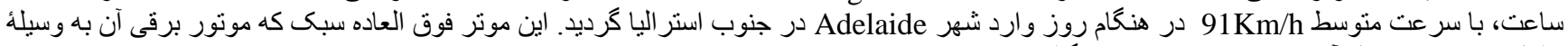

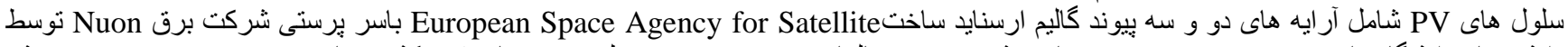

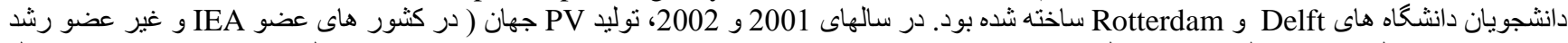

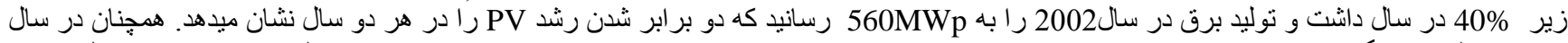

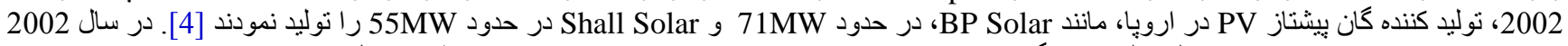

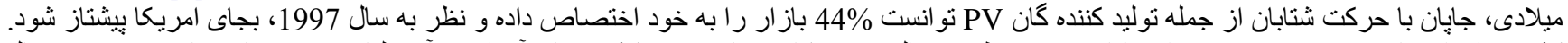

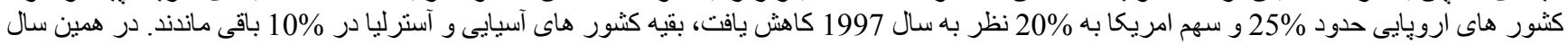

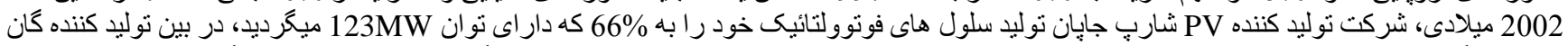

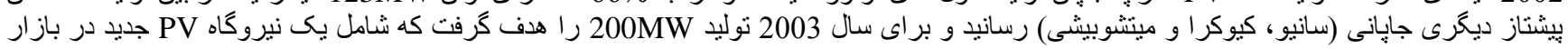

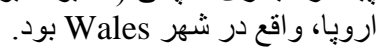

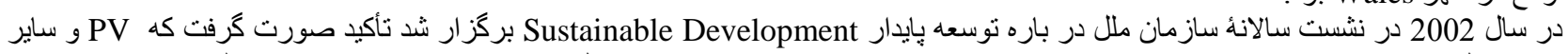

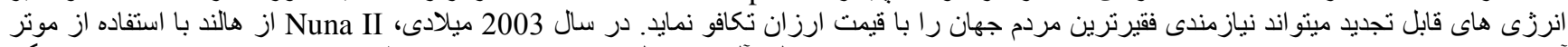

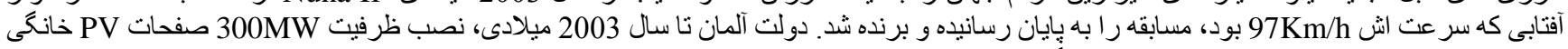

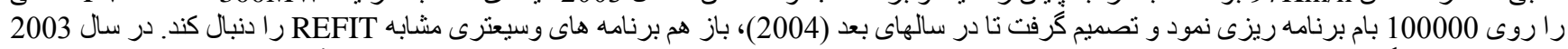

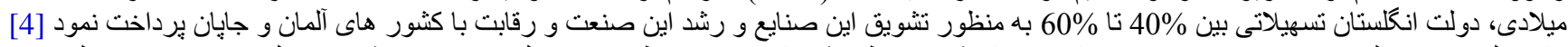

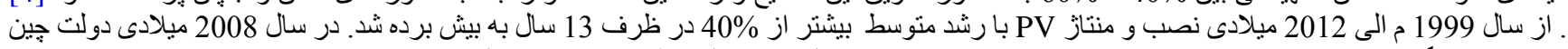

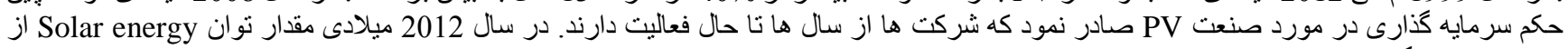

جدول 1. نوان توليدى برق آفنتابى در جهان از سال 2005 الى 2015 ميلادى به كيكا وات.

حد100GWp كذنشت

\begin{tabular}{l|l|l|l|l|l|l|l|l|l|l}
\hline $\mathbf{2 0 1 5}$ & $\mathbf{2 0 1 4}$ & $\mathbf{2 0 1 3}$ & $\mathbf{2 0 1 2}$ & $\mathbf{2 0 1 1}$ & $\mathbf{2 0 1 0}$ & $\mathbf{2 0 0 9}$ & $\mathbf{2 0 0 8}$ & $\mathbf{2 0 0 7}$ & $\mathbf{2 0 0 6}$ & $\mathbf{2 0 0 5}$ \\
\hline 227 & 177 & 138 & 100 & 70 & 40 & 23 & 16 & 9 & 6.7 & 5.1 \\
\hline
\end{tabular}

4

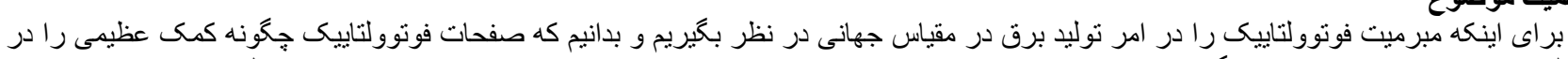

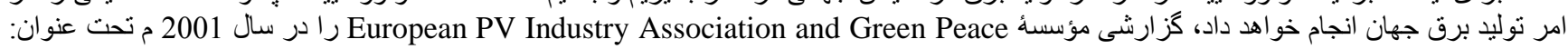
برق خورشيدى بر ایى بيش از يكى مليارد جمعيت و دو مليون شئ شغل تا سال 2020 م در نظر است تعداد يك مليارد PV مستقل از شبكهو 82 ميليون متصل به 


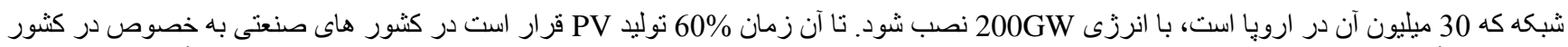

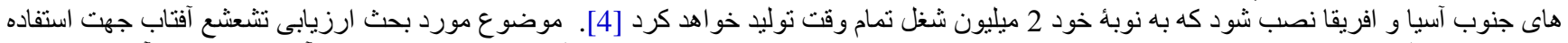

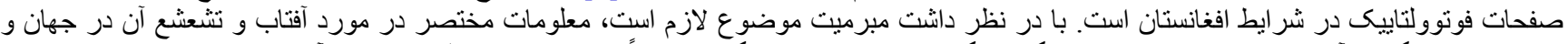

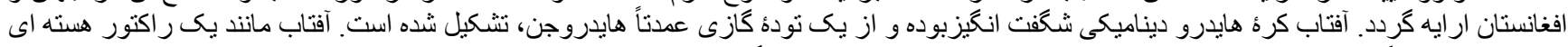

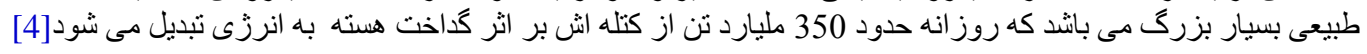

5

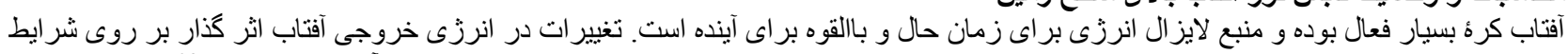

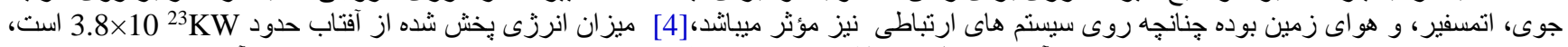

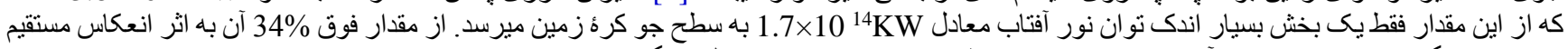

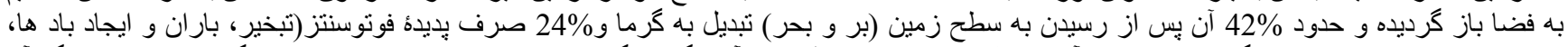

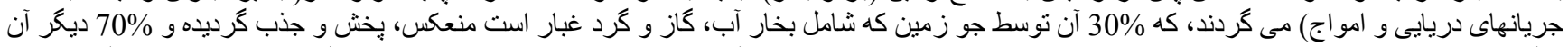

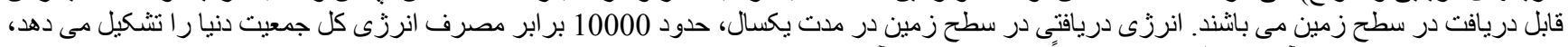

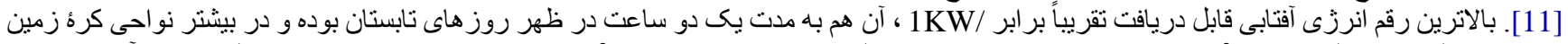

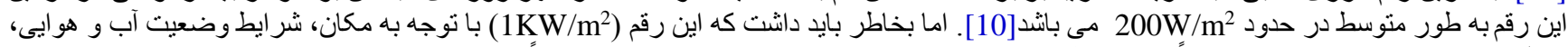

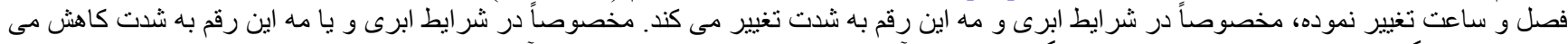

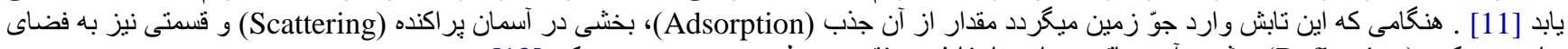

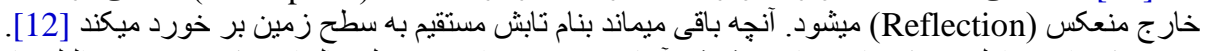

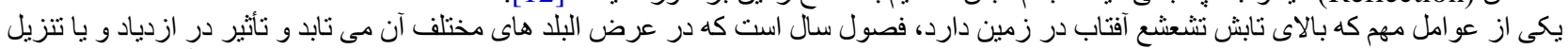

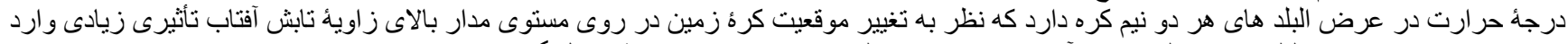

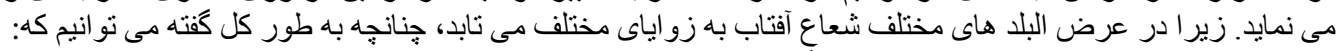

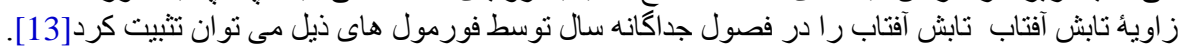

- Latitude

(Latitude + $\left.23^{\circ} 30^{\prime}\right)$

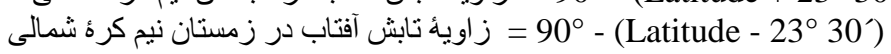

جون موضوع بحث ما ارزيابى تشعشع آفتاب در شر ايط افغانستان است. بناءً لازم است تأ موقعيت جغر افيايى افغانستان را أتثبيت نمائيم:

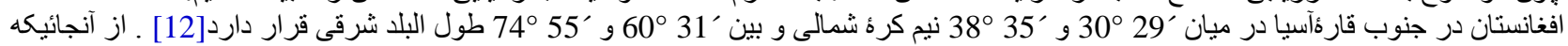

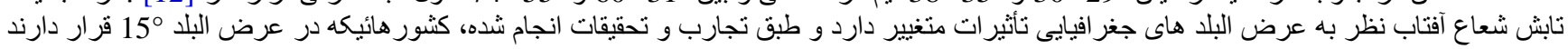

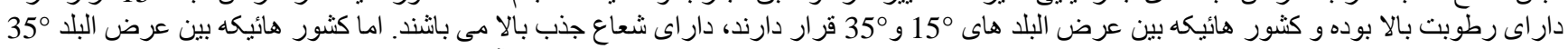

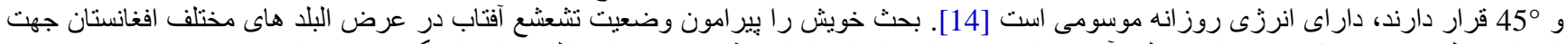

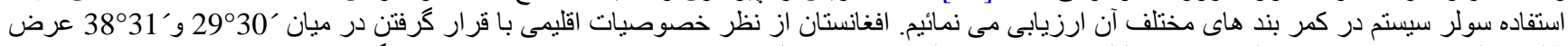

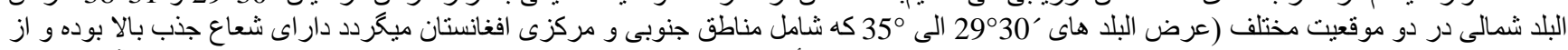

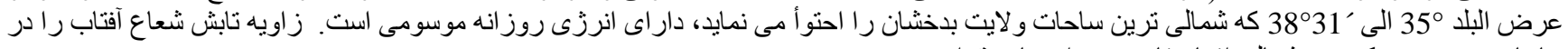

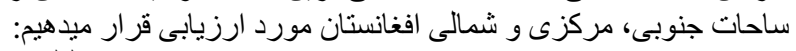

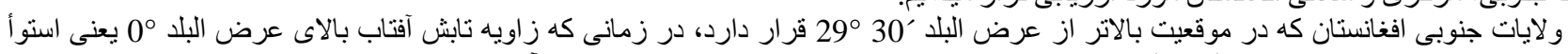

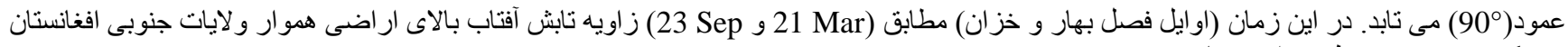

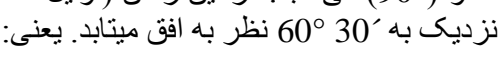
$90^{\circ}-29^{\circ} 30^{\prime}=60^{\circ} 30^{\prime}$

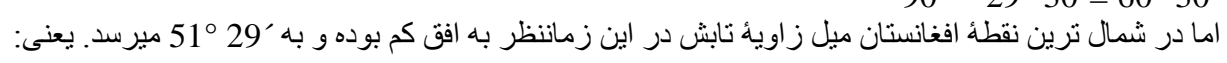
$90^{\circ}-38^{\circ} 31^{\prime}=51^{\circ} 29^{\prime}$

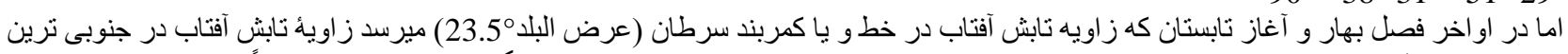

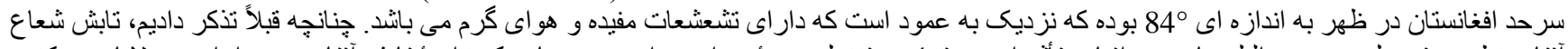

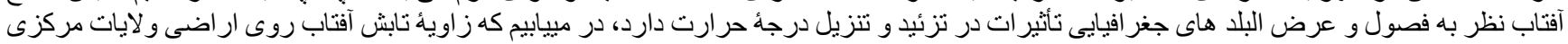

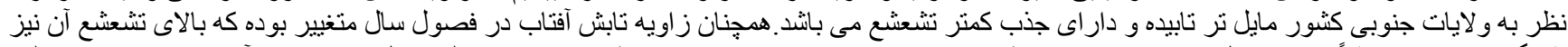

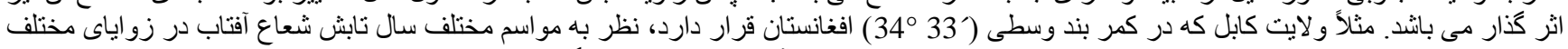

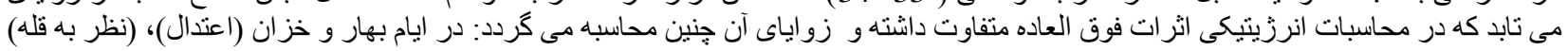
$90^{\circ}-34^{\circ} 33^{\prime}=55^{\circ} 27^{\prime}$

در ايام انقلاب شمسى تابستان (نظر به قله $90^{\circ}-\left[34^{\circ} 33^{\prime}+23^{\circ} 30^{\prime}\right]=90^{\circ}-58^{\circ} 3^{\prime}=31^{\circ} 57^{\prime}$ در ايام انقلاب شمسى زمستان (نظر به قله

$90^{\circ}-\left[34^{\circ} 33^{\prime}-23^{\circ} 30^{\prime}\right]=90^{\circ}-11^{\circ} 3^{\prime}=78^{\circ} 57^{\prime}$

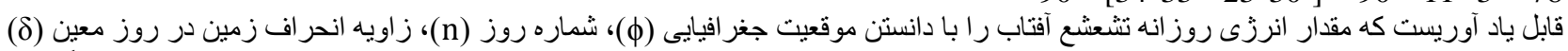

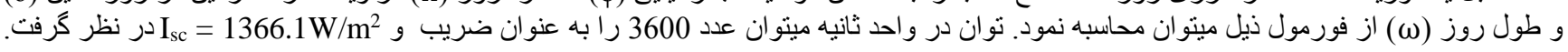

$$
\mathrm{H}_{\mathrm{o}}=\mathrm{I}_{\mathrm{sc}} \frac{24(3600)}{\pi}\left[1+0.033 \operatorname{Cos} \frac{360 \mathrm{n}}{365}\right]\left[\frac{\pi \omega}{180} \operatorname{Sin} \phi \operatorname{Sin} \delta+\operatorname{Cos} \phi \operatorname{Cos} \delta \sin \omega\right]
$$

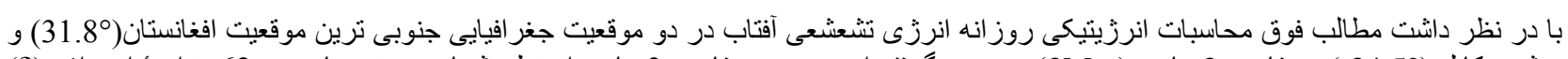

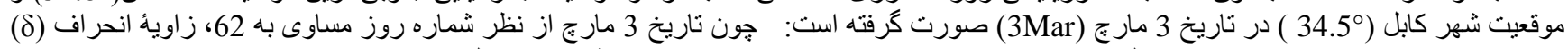

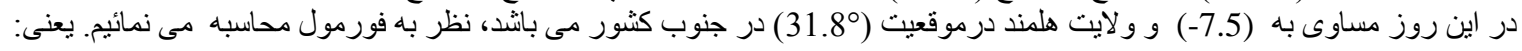


$\delta=(-7.5) \& \phi={ }^{\circ} 31.8$

$\omega=\operatorname{Cos}^{-1}(-\tan \delta \tan \phi)$

$\omega=\operatorname{Cos}^{-1}\left[-\tan (-7.5) \tan \left(31.8^{\circ}\right)\right]=\operatorname{Cos}^{-1}[-(-0.13165)(0.62)]=\operatorname{Cos}^{-1}(0.0816)$

$\omega=85.31^{\circ}$

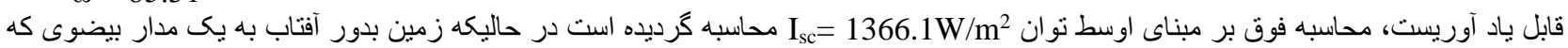

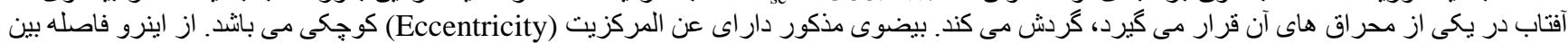

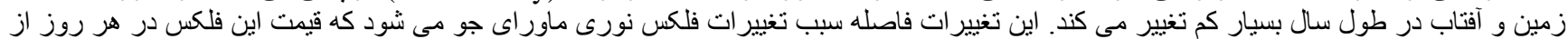

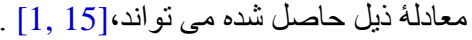

$\mathrm{I}_{\mathrm{sc}}=\mathrm{I}_{\mathrm{sc}}\left(1+0.033 \operatorname{Cos} \frac{360 \times \mathrm{n}}{365}\right)(3)$

$\mathrm{I}_{\mathrm{sc}}=1366.1\left(1+0.033 \operatorname{Cos} \frac{360 \times 62}{365}\right)=1366.1(1+0.033 \operatorname{Cos} 61.15)$

$=1366.1[1+0.033(0.4825)]$

$\mathrm{I}_{\mathrm{sc}}=1387.852 \mathrm{~W} / \mathrm{m}^{2}$

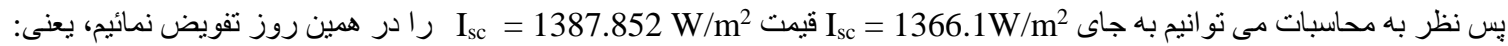

$\mathrm{H}_{\mathrm{o}}=(1387.8) \mathrm{W} / \mathrm{sec} \frac{24(3600)}{\pi}\left[1+0.033 \operatorname{Cos} \frac{360 \times 62}{365}\right]\left[\frac{\pi 85.31}{180} \operatorname{Sin}(31.8) \operatorname{Sin}(-7.5)+\operatorname{Cos}(31.8) \operatorname{Cos}(-7.5) \operatorname{Sin}\left(85.31^{\circ}\right)\right]$

$=38167239.7[(1+0.0159227)][(1.48894)(0.52)(-0.13)+(0.849)(0.99)(0.996)]$

$=38167239.7[1.0159227][-0.10065+0.8376]$

$=28575598 \mathrm{~J}=28.6 \mathrm{MJ} / \mathrm{m}^{2}$

بناءً نظر به محاسبه فوق كفته مى تو انيم كه مقدار انرزى نشعشع آفتاب در تاريخ 3 مارعج (3Mar) در موقعيت (31.8) جنوب افغانستان به مقياس جهانى

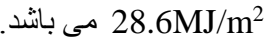
حال هركاه خو استه باثشيم مقدار اوسط انرزى تشعشع آفتاب را در تاريخ 3 مار ج (3Mar) در شهر كابل محاسبه نمائيم، داريم كه: $\mathrm{n}=62, \quad \delta=(-7.5) \& \phi=34.5^{\circ}$

$\mathrm{H}_{\mathrm{o}}=\mathrm{I}_{\mathrm{sc}} \frac{24(3600)}{\pi}\left[1+0.033 \operatorname{Cos} \frac{360 \mathrm{n}}{365}\right]\left[\frac{\pi \omega}{180} \operatorname{Sin} \phi \operatorname{Sin} \delta+\operatorname{Cos} \phi \operatorname{Cos} \delta \operatorname{Sin} \omega\right]$ جون در فورمول ذيل داريم

$\omega=\operatorname{Cos}^{-1}(-\tan \delta \tan \phi)$

دانستن ف نيز ضروريست. بناءً اولاً $\omega$ را محاسبه و در فورمول وضع مينمائيح.

$\omega=\operatorname{Cos}^{-1}\left[-\tan (-7.5) \tan \left(34.5^{\circ}\right)\right]=\operatorname{Cos}^{-1}[-(-0.13165)(0.687)]=\operatorname{Cos}^{-1}(0.09048)$

$\omega=84.8^{\circ}$

$\mathrm{H}_{\mathrm{o}}=(1387.8) \frac{24(3600)}{\pi}\left[1+0.033 \operatorname{Cos} \frac{360 \times 62}{365}\right]\left[\frac{\pi 84.8}{180} \operatorname{Sin}(34.5) \operatorname{Sin}(-7.5)+\operatorname{Cos}(34.5) \operatorname{Cos}(-7.5) \operatorname{Sin}\left(84.8^{\circ}\right)\right]$

$=38167239.7[(1+0.0159227)][(1.48)(0.57)(-0.13)+(0.82)(0.99)(0.996)]$

$=38167239.7[1.0159227][-0.109+0.808]=27103700 \mathrm{~J}=27.1 \mathrm{MJ} / \mathrm{m}^{2}$

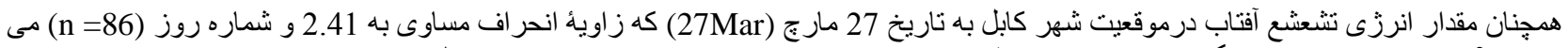

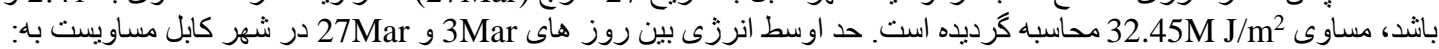

$\left(27.1 \mathrm{MJ} / \mathrm{m}^{2}+32.45 \mathrm{MJ} / \mathrm{m}^{2}\right) / 2=29.77 \mathrm{MJ} / \mathrm{m}^{2}$

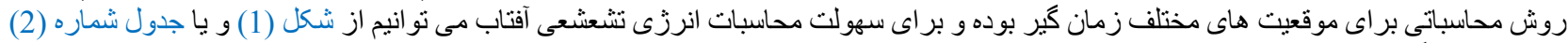

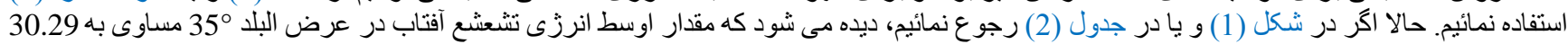

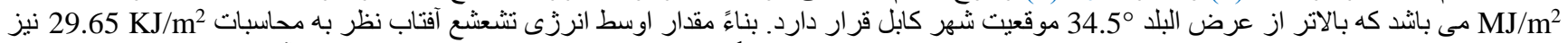

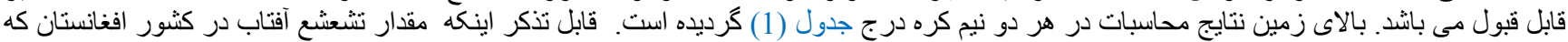

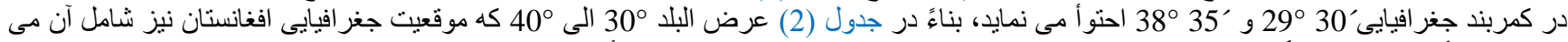

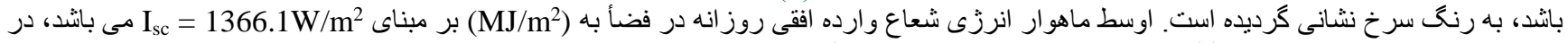

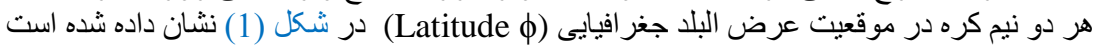

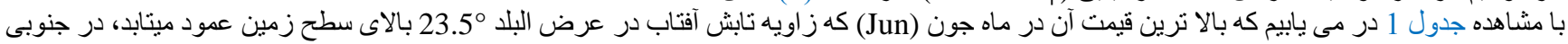

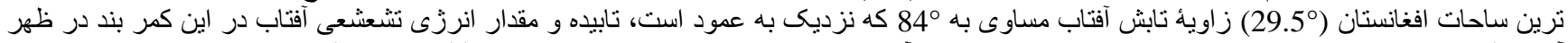

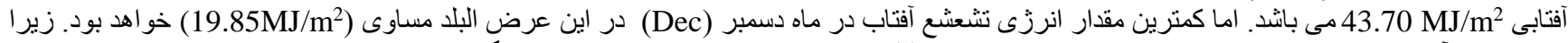

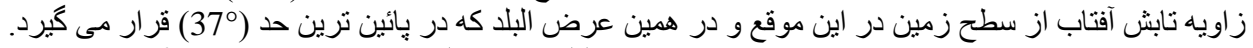

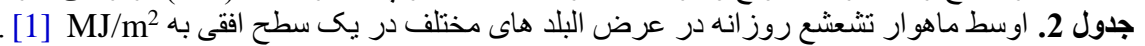

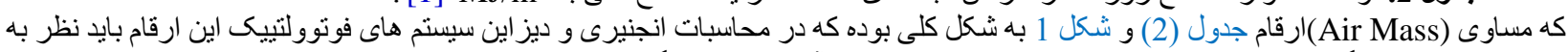

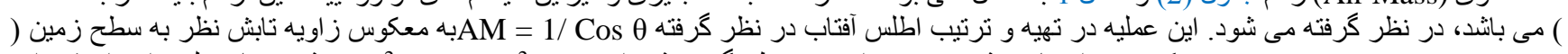

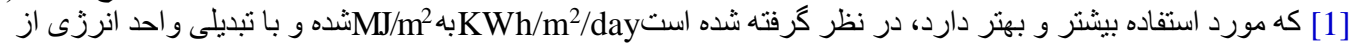


Solar Resource

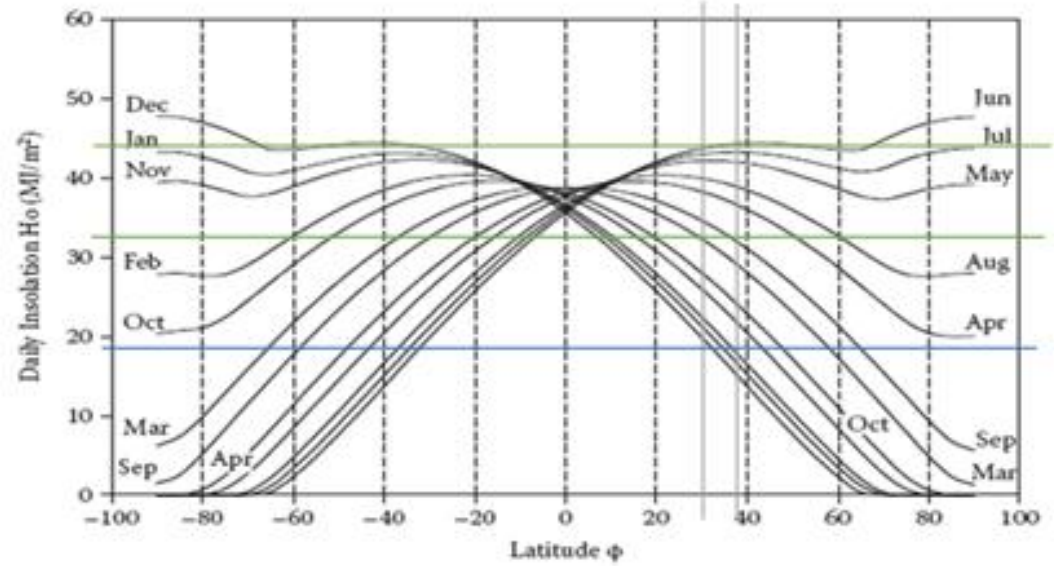

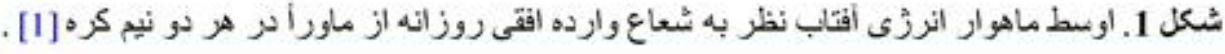

\begin{tabular}{|c|c|c|c|c|c|c|c|c|c|c|c|c|}
\hline \multirow{2}{*}{ هرض البلد } & \multicolumn{12}{|c|}{ ماه هاى سال (Month of year) } \\
\hline & Jan & $\mathrm{Feb}$ & Mar & Apr & May & Jun & Jul & Agu & Sep & Oct & Nov & Dec \\
\hline-90 & 43.31 & 27.82 & 6.2 & 0 & 0 & 0 & 0 & 0 & 1.38 & 20.36 & 39.41 & 47.76 \\
\hline-85 & 43.21 & 27.96 & 7.35 & 0.01 & 0 & 0 & 0 & 0 & 2.51 & 20.75 & 39.62 & 47.64 \\
\hline-80 & 42.63 & 27.64 & 9.85 & 0.67 & 0 & 0 & 0 & 0.04 & 5.18 & 21.09 & 39.1 & 46.98 \\
\hline-75 & 41.81 & 27.82 & 12.86 & 2.49 & 0 & 0 & 0 & 0.8 & 8.32 & 22.41 & 38.35 & 46.08 \\
\hline-70 & 40.74 & 29.07 & 15.9 & 5.22 & 0.42 & 0 & 0.04 & 2.76 & 11.5 & 24.54 & 37.66 & 44.83 \\
\hline-65 & 40.43 & 30.84 & 18.87 & 8.23 & 2.16 & 0.37 & 1.1 & 5.47 & 14.63 & 26.82 & 38.02 & 43.6 \\
\hline-60 & 40.97 & 32.67 & 21.71 & 11.32 & 4.7 & 2.29 & 3.34 & 8.42 & 17.67 & 29.06 & 38.93 & 43.59 \\
\hline-55 & 41.66 & 34.41 & 24.4 & 14.4 & 7.54 & 4.82 & 6.04 & 11.47 & 20.58 & 31.16 & 39.9 & 43.89 \\
\hline-50 & 42.29 & 35.98 & 26.92 & 17.42 & 10.54 & 7.66 & 8.96 & 14.53 & 23.35 & 33.09 & 40.78 & 44.19 \\
\hline-45 & 42.78 & 37.35 & 29.23 & 20.34 & 13.59 & 10.65 & 12 & 17.55 & 25.94 & 34.79 & 41.5 & 44.37 \\
\hline-40 & 43.07 & 38.43 & 31.33 & 23.14 & 16.65 & 13.72 & 15.07 & 20.48 & 28.35 & 36.26 & 42 & 44.38 \\
\hline-35 & 43.12 & 39.36 & 33.2 & 25.78 & 19.65 & 16.81 & 18.12 & 23.29 & 30.54 & 37.48 & 42.26 & 44.16 \\
\hline-30 & 42.92 & 39.97 & 34.81 & 28.24 & 22.56 & 19.86 & 21.12 & 25.96 & 32.5 & 38.43 & 42.26 & 43.71 \\
\hline-25 & 42.46 & 40.3 & 36.17 & 30.5 & 25.36 & 22.84 & 24.02 & 28.46 & 34.21 & 39.11 & 42 & 42.99 \\
\hline-20 & 41.73 & 40.35 & 37.25 & 32.54 & 28.01 & 25.72 & 26.79 & 30.77 & 35.67 & 39.49 & 41.46 & 42.02 \\
\hline-15 & 40.73 & 40.11 & 38.04 & 34.35 & 30.48 & 28.46 & 29.42 & 32.86 & 36.85 & 39.59 & 40.64 & 40.8 \\
\hline-10 & 39.47 & 39.59 & 38.55 & 35.9 & 32.76 & 31.03 & 31.85 & 34.72 & 37.76 & 39.4 & 39.56 & 39.31 \\
\hline-5 & 37.45 & 38.78 & 38.77 & 37.19 & 34.82 & 33.43 & 34.09 & 36.33 & 38.38 & 38.92 & 38.21 & 37.58 \\
\hline 0 & 36.18 & 37.69 & 38.7 & 38.2 & 36.65 & 35.61 & 36.12 & 37.69 & 38.71 & 38.16 & 36.6 & 35.61 \\
\hline 5 & 34.18 & 36.33 & 38.33 & 38.94 & 38.24 & 37.58 & 37.91 & 38.77 & 38.75 & 37.11 & 34.75 & 33.42 \\
\hline 10 & 31.95 & 34.72 & 37.67 & 39.38 & 39.57 & 39.31 & 39.46 & 39.58 & 38.5 & 35.79 & 32.66 & 31.03 \\
\hline 15 & 29.53 & 32.85 & 36.73 & 39.54 & 40.63 & 40.79 & 40.74 & 40.1 & 37.95 & 34.21 & 30.37 & 28.45 \\
\hline 20 & 26.93 & 30.76 & 35.51 & 39.4 & 41.42 & 42.02 & 41.77 & 40.34 & 37.11 & 32.38 & 27.88 & 25.71 \\
\hline 25 & 24.17 & 28.45 & 34.02 & 38.98 & 41.93 & 42.99 & 42.52 & 40.29 & 36 & 30.31 & 25.22 & 22.3 \\
\hline 30 & 21.28 & 25.95 & 32.28 & 38.27 & 42.17 & 43.7 & 43.01 & 39.95 & 34.61 & 28.03 & 22.41 & 19.85 \\
\hline
\end{tabular}




\begin{tabular}{l|l|l|l|l|l|l|l|l|l|l|l|l}
35 & 18.29 & 23.28 & 30.29 & 37.29 & 42.14 & 44.15 & 43.24 & 39.34 & 32.96 & 25.55 & 19.49 & 16.8 \\
\hline 40 & 15.28 & 20.46 & 28.07 & 36.04 & 41.85 & 44.37 & 43.22 & 38.46 & 31.07 & 22.89 & 16.48 & 13.71 \\
\hline 45 & 12.17 & 17.52 & 25.65 & 34.53 & 41.32 & 44.36 & 42.96 & 37.33 & 28.94 & 20.08 & 13.42 & 10.64 \\
\hline 50 & 9.14 & 14.5 & 23.03 & 32.79 & 40.58 & 44.17 & 42.51 & 35.96 & 26.59 & 17.14 & 10.37 & 7.64 \\
\hline 55 & 6.2 & 11.44 & 20.25 & 30.83 & 39.66 & 43.87 & 41.91 & 34.39 & 24.05 & 14.12 & 7.38 & 4.81 \\
\hline 60 & 3.48 & 8.39 & 17.32 & 28.69 & 38.65 & 43.57 & 41.26 & 32.65 & 21.33 & 11.04 & 4.54 & 2.28 \\
\hline 65 & 1.2 & 5.43 & 14.27 & 26.42 & 37.7 & 43.57 & 40.78 & 30.82 & 18.46 & 7.96 & 2.03 & 0.36 \\
\hline 70 & 0.06 & 2.71 & 11.14 & 24.08 & 37.27 & 44.8 & 41.19 & 19.05 & 15.47 & 4.98 & 0.35 & 0 \\
\hline 75 & 0 & 0.73 & 7.97 & 21.87 & 37.87 & 46.05 & 42.3 & 27.83 & 12.41 & 2.31 & 0 & 0 \\
\hline 80 & 0 & 0.02 & 4.86 & 20.43 & 38.61 & 46.95 & 43.12 & 27.6 & 9.35 & 0.06 & 0 & 0 \\
\hline 85 & 0 & 0 & 2.29 & 20.01 & 39.06 & 47.49 & 43.62 & 27.87 & 6.73 & 0.01 & 0 & 0 \\
\hline 90 & 0 & 0 & 1.25 & 20.07 & 39.21 & 47.67 & 43.79 & 27.98 & 5.66 & 0 & 0 & 0 \\
\hline
\end{tabular}

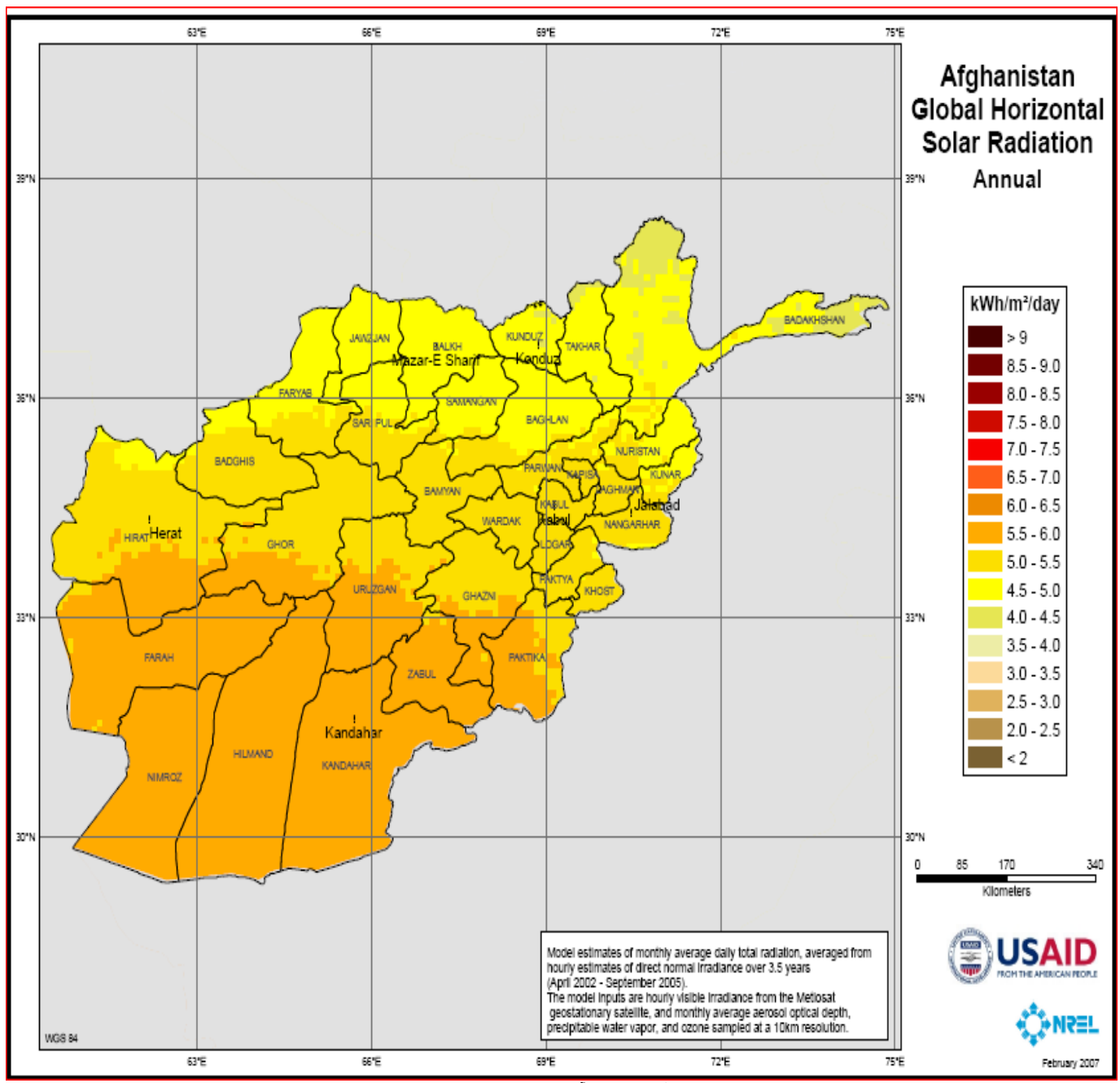

شُكل 2. اطلس تشعشع آفتاب در افغانستان (NREL)

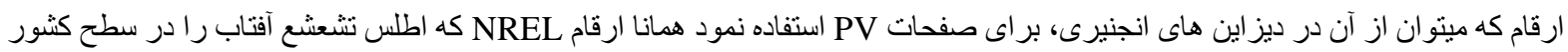

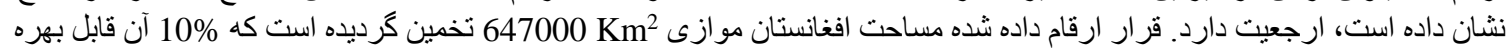

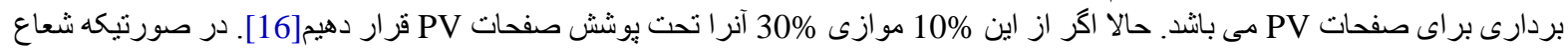

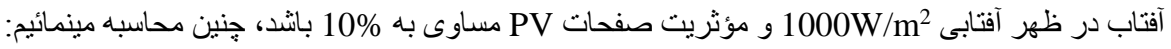

(647000Km²) (0.1) = مساحت قابل بوشش بر اي صفحات فوتوولتاييك 
$\mathrm{P}_{(\mathrm{MW})}=\left(19410 \mathrm{Km}^{2}\right)\left(1000000 \mathrm{~m}^{2} / \mathrm{Km}^{2}\right)\left(700 \mathrm{~W} / \mathrm{m}^{2}\right)(0.1$ penal eff $)=13548700 \mathrm{MW}$

اطلس تشعشع آفتاب با مقدار اوسط سالانه تشعشع آفتاب به مقياس (KWh/m²/day) در كمربند هاى جغر افيايى افغانستان كه از طرف

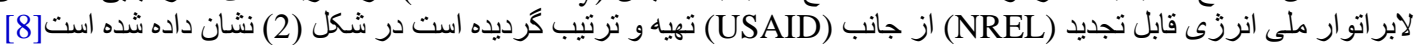

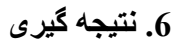

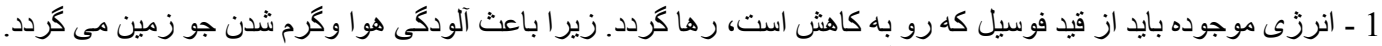

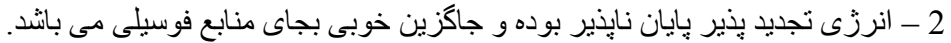

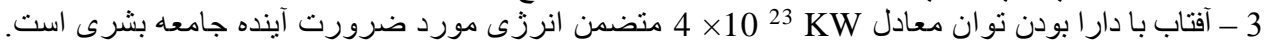

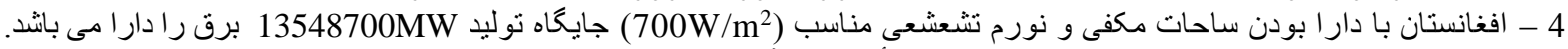

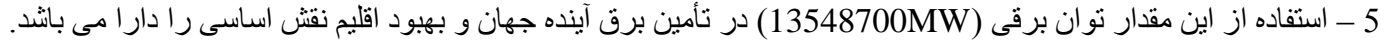
1

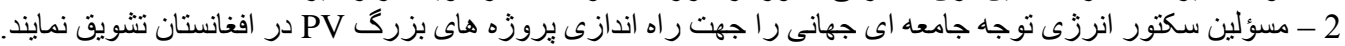

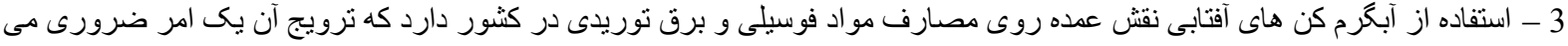

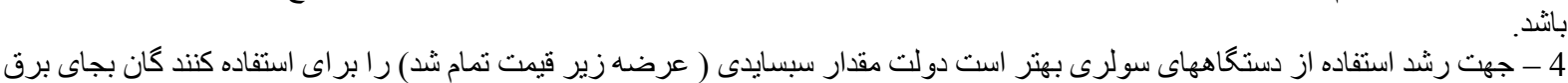

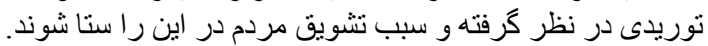

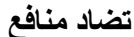

نويسنده تعهر مى نمايد كه مقاله تضاد منافع ندارد.

\section{References}

[1] Dalkey, Earth in space (Solar System), 3rd ed. vol. 192: Hamoon Summer, 2010.

[2] Tafreshi, Sources of electricity production in the 21st century: Khajeh Nasir al-Din Tusi University of Technology Publications, 2007.

[3] Tafreshi, "Sources of electricity production in the 21st century," ed Khajeh Nasir al-Din Tusi University of Technology Publications, 2007, p. 602.

[4] F. B. Gaddy, New energies: Energy for a sustainable future. Translator Partovi_Abdar Rahim: University of Tehran Press, 2007.

[5] N. Kaabi, Energy saving with solar batteries: Fuel Consumption Optimization Organization, 2013.

[6] K.-D. Jäger, O. Isabella, A. H. Smets, R. A. van Swaaij, and M. Zeman, Solar energy: Fundamentals, technology and systems: UIT Cambridge, 2016.

[7] Kokabi, Solar energy, principles of thermal collection and storage: Saboun Printing House, 2014.

[8] K. Alizadeh and A. Gholam, Weather and climatology AH, 381, 6th ed.: Ferdowsi University of Mashhad, 2004

[9] K. Alizadeh and A. Gholam, Weather and climatology, 6th ed.: Ferdowsi University of Mashhad, 2004.

[10] Ariz, Afghanistan climate, faculty of literature and humanities vol. 95: Kabul University, 1348.

[11] Ariz, General geography of Afghanistan: Kabul University Press, 1362.

[12] Hosseini, wind energy and its role in solving the problem of electricity in the country vol. 179: Academy of Sciences, 2016.

[13] Araz, Climatology, faculty of literature and humanities vol. 157: Kabul University, 1357.

[14] V. L. A. Kristoferson and R. Bokalders, "Renewable energy technologies, their application in developing countries," p. 319, 1986.

[15] K. Alizadeh and A. Gholam, "Weather and climatology, Ferdowsi University of Mashhad." vol. 381, 6th ed: Summer, 2004.

[16] Sajjadifar, Recognition of space and solar system, published by Hamoon: Winter, 2008. 